\title{
Comparison of Effect of One Course of Intense Exercise (Wingate test) on Serum Levels of Interleukin-17 in Different Groups of Athletes
}

\author{
Asghar Tofighee ${ }^{1}$; Hossein Ali Khazaei ${ }^{2, *}$; Arman Jalili $^{3}$ \\ ${ }^{1}$ Department of Sport Physiology, Urmia University, Urmia, IR Iran \\ ${ }^{2}$ Research Center for Children and Adolescents Health, Department of Medical Immunology and Hematology, Zahedan University of Medical Sciences, Zahedan, IR Iran \\ ${ }^{3}$ Department of Sport Physiology, Tehran University, Tehran, IR Iran \\ *Corresponding author: Hossein Ali Khazaei, Research Center for Children and Adolescents Health, Department of Medical Immunology, Zahedan University of Medical Sciences, \\ Zahedan, IR Iran. Tel: +98-9153403094, Fax:+98-5433295563, E-mail: h_khazaei118@yahoo.com
}

Received: July 22, 2013; Accepted: December 27, 2013

\begin{abstract}
Background: Research on the effects of exercise on immune function, has a wide range of sporting activities. Study on the long-term effects of regular exercise on serum levels of cytokines such as interleukin-17 have shown that moderate and regular exercise, has an important role in the prevention and treatment of many diseases.

Objectives: Exhaustive exercise has a deep effect on cellular, humoral, innate immunity and the amount of cytokines of an athlete's immune system. So this study was designed to compare the effect of one course of exhaustive exercise on serum levels of interleukin (IL)-17 in different groups of athletes.

Patients and Methods: Forty professional athletes with a mean age of $25.1 \pm 5.0$ years, divided equally in 4 groups (handball, volleyball, Sepak-takraw and climbing) were selected for this purpose. 30 second Wingate test for each athlete was used to assess anaerobic power. Blood samples before, immediately after and 2 hours after exercise was collected and the amount of serum IL-17 was measured.

Results: The results showed that the level of IL-17 in the study groups before and after the two hours exercise did not significantly change in all four groups.
\end{abstract}

Conclusions: The results showed that short anaerobic exercise has no effect on the level of IL-17.

Keywords:Exercise; Interleukin-17; Exercise Test

\section{Background}

The human body is constantly under the influence of an environment full of infectious agents. These microorganism, have the potential roles for spreading uncontrolled materials, causing pathological damage and eventually destruction of their host (1). In the recent years, studies have shown the role of physical stress on the immune system. These studies have examined the effects of exercise on immune function $(2,3)$. Some of them have shown that moderate and regular exercise, has an important role in the prevention and treatment of many diseases $(4,5)$.

Research on the effects of exercise on immune function, encompasses a wide range of sporting activities including short-term, exhaustive activity, endurance and longterm activities, and regular light exercise. Study on the long-term effects of regular exercise on immune function is used extensively in the development of public health and prevention among nations in which their active elderly population is increasing $(6,7)$.

Cytokines are comprised of a group of proteins, soluble proteins or glycol proteins that perform the role of message transmission between immune cells and other cells, and can have different effects on growth and acti- vation of normal and tumor cells. Cytokines also have anti-inflammatory, pro-inflammatory and suppressive activities on the immune system in various conditions (8). Studies have shown that vigorous and hard physical activity, causes inflammation and secretion pro-inflammatory cytokines such as interleukin (IL)-6, IL-1 and tumor necrosis factor-alpha (TNF- $\alpha$ ) (9-11). So an intense training session has a significant impact on the balance of cytokine active and may cause a change in the distribution of cells and immune system will be suppressed and weakened temporarily (9). IL-17 is a proinflammatory cytokine, which is expressed in different cells, including lymphocytes and microglia cells (12-14). IL-17 is also an inflammatory guidance cytokine. Several isoforms of the receptors of this cytokine have been identified from different areas of the body. This suggests that the IL-17 function can be varied and complex as has been reported. This cytokine increases in inflammatory diseases such as asthma, prostate cancer and inflammatory disorders of the joints (15). IL-17 production is done by TH17 cells and plays a major role in autoimmune inflammation, multiple sclerosis, rheumatoid arthritis and diabetes (16). Indirect effects of IL-17 cause produc- 
tion of cytokines such as IL-10, IL-8, IL-6 and IL-4. Many of these cytokines are produced during strenuous exercise. IL-17 often acts as an intracellular messenger molecule after binding to the target cell, which will initiate many biological activities. Evidence suggests that IL-17 can be considered as booster, accumulator and activator of neutrophils in the lung, joint space, central nervous system and intestinal tissue (17).

Some researchers believe that plasma levels of IL-17 may be a useful biochemical marker for acute inflammation that is produced in skeletal muscle (18). This cytokine is able to activate macrophages, fibroblasts, secretion of cytokines IL-6, IL-1, IL-8, secretion of prostaglandin E2, nitric oxide, CPK and CRP (18). On the other hand, some physical activity (especially extrovert, sudden and severe) may lead to muscle damage. Muscle damage caused by exercise starts with the mechanical contraction of muscle and this causes production of inflammatory mediators such as cytokines and chemokines leading to movement of neutrophils into the circulation. Neutrophils enter the muscle tissue from the blood flow and due to phagocytosis, the muscle is damaged. Research shows that exercise causes many changes in the components of the immune system, including increased levels of IL-6, IL-1 and TNF- $\alpha$ (19). Chang et al. (20) in the research on cardiovascular patients found that the effect of IL-17 in stimulating C-reactive protein (CRP) is independent of IL- 6 in the smooth muscle cells of the heart and at the same time IL- 6 reinforces the effects of IL-17. The impact of the amount of training on IL-17 in some studies as a factor has been considered. Duzova et al. examined 2 running programs on a treadmill, severe (speed $85 \%$ maximal oxygen consumption with slope) and light (no gradient and with 50 to $60 \%$ of maximum oxygen consumption). They found that after 8 weeks, the levels of these cytokines were increased in the group that used intensive training, but no change was observed in the other group (21). Golzari et al. found in a study that an 8-week combined program including warm-up, stretching exercises, strength exercises, aerobic exercise and a relaxation program is used in the two sessions, level of IL-17 will not increase and in some cases there is even a reduction because of low levels of exercise intensity (22). The researchers also stated that 12 weeks of intense physical activity increased IL-17. But no change was observed in the control group and the group that had moderate activity (21).

\section{Objectives}

Given the conflicting research on the effects of exercise on cytokine concentrations and the lack of studies about the effects of short-term anaerobic exercise on various athletes and also lack of research on the effect of exercise on serum IL-17, led us to assess the pro-inflammatory cytokine profile of IL-17 followed by a session of vigorous activity.

\section{Patients and Methods}

This research is a semi-experimental study and approved by the Ethics Committee of Urmia University. The study population included all national athletes in the premier league in the field of handball, volleyball, Sepak-Takraw and climbing in the city of Zahedan. The participants were invited to deliberate after taking informed consent from all participants. In each field of sport, 10 adults ( 40 people in total) between $20-25$ years old with a mean age of $25.1 \pm 5.0$ years were selected. We used an exhaustive exercise plan (Wingate) using a bicycle ergometer Munark in 1 session). Each session lasted one hour and 30 minutes and included a group of warms-ups first, then the main exercise and cool down at the end of each session. The Wingate test is maximal work on a bicycle ergometer for 30 seconds, which measures the lower body anaerobic performance. Before each test, all four athlete groups were warmed for 5 minutes by stress-free warm-up method on the bike, and after doing this test, cooled for 1 to 2 minutes. Blood samples from both groups before the start of each session, and immediately after each exercise session, and two hours after training, were collected and transferred to the laboratory for testing. Measurement of IL-17 serum concentration (picogram in $\mathrm{mL} \mathrm{pg/mL}$ ) was done by ELISA method using commercial label BENDERMED kit (BIOSCINCE-UK).

Results from blood samples were analyzed by SPSS version 18 software. Descriptive statistics method was used for the classification and adjustment of the data and determination of central index (mean and standard deviation of the dispersion index). To determine the normal distribution of data, Kolmogorov-Smirnov (KS) exam was used. For comparison of pre-and post-tests for withingroup, repeated measures test was used. For comparison between groups, one way ANOVA test was also used. For determination of any statistical correlation, P values were measured and the result of less than 0.05 was considered as a significant correlation between four groups of athletes.

\section{Results}

The total demographic data of four groups of studied athletes are illustrated in Table 1 . These parameters failed to show any differences between basic physiological data and different groups of athletes. The mean serum level of IL-17 in all participants before starting sport $(119.0 \pm 60.4 \mathrm{pg} / \mathrm{mL})$ was lower than amounts of this cytokine in both a little and 2 hour after exercise $(127.3 \pm 26.9 \mathrm{pg} / \mathrm{mL}$ and $129.0 \pm 45.2 \mathrm{pg} / \mathrm{mL}$ respectively however this variation was not statistically significant ( $\mathrm{P}=0.8$ and 0.7 respectively). The results of three times probability IL-17 level used in the study showed no statistical correlation in the amount of this cytokine in four groups of athletes before, after and 2 hours after exercise $(\mathrm{P}>0.05)$ (Table 2$)$. 
Tofighee A et al.

\begin{tabular}{lcccc}
\hline Table 1. The Result of Age, Weight, High, and BMI of Four Groups of Athletes a,b & & \\
\hline Types of Sport & Age, $\mathbf{y}$ & BMI, $\mathbf{~ k g} / \mathbf{m}^{2} \mathrm{~b}$ & Height, $\mathbf{~ m ~}$ & Weight, kg \\
\hline Sepak-takraw & $22.5 \pm 5.1$ & $21.4 \pm 2.2$ & $182.1 \pm 6.2$ & $69.6 \pm 4.7$ \\
Climbing & $26.9 \pm 4.3$ & $23.2 \pm 2.1$ & $176.1 \pm 5.3$ & $71.7 \pm 5.2$ \\
Volleyball & $24.7 \pm 3.4$ & $22.8 \pm 1.3$ & $188.4 \pm 4.3$ & $78.2 \pm 5.2$ \\
Handball & $22.1 \pm 3.1$ & $23.1 \pm 1.1$ & $180.5 \pm 3.3$ & $76.9 \pm 2.8$ \\
\hline
\end{tabular}

a Abbreviation: BMI, Body mass index.

$\mathrm{b}$ Data are presented as mean $\pm \mathrm{SD}$.

Table 2. The Results of Three Times Probability Interleukin-17 Level Used (pg/mL) in Four Groups of Athletes Before, After and 2 Hours After Exercise a

\begin{tabular}{lcccc}
\hline Types of Sport & Before Exercise & After Exercise & 2 Hours After Exercise & PValue \\
\hline Sepak-Takraw & $122.7 \pm 25.3$ & $124.8 \pm 22.3$ & $117.4 \pm 29.4$ & 0.8 \\
Climbing & $119.2 \pm 17.5$ & $116.1 \pm 36.5$ & $124.1 \pm 21.4$ & 0.9 \\
Volleyball & $126.5 \pm 23.6$ & $126.6 \pm 25.9$ & $131.8 \pm 13.4$ & 0.8 \\
Handball & $123.7 \pm 21.2$ & $130.7 \pm 33.7$ & $131.4 \pm 33.3$ & 0.7 \\
\hline
\end{tabular}

${ }^{\mathrm{a}}$ Data are presented as mean \pm SD.

\section{Discussion}

The results of this study indicate that the amount of IL17 in all four groups of studied athletes has no significant change compared to pre-exercise. It is also indicative that training intensity had no significant impact on the IL-17 before, after, and 2 hour after exercise, which is considered as an important factor in the investigation. Some researchers have suggested that increased exercise intensity, has changed the level of this cytokine (21). This mechanism is probably related to the issue of strenuous exercise causing the release of proinflammatory cytokines and anti-inflammatory cytokines by peripheral blood leukocytes and skeletal muscle $(18,19,21)$. According to various studies the intensity or duration of exercise is a major factor in the increased production of IL-17. When this research was of appropriate intensity, this practice seems to have no effect, because the time was short. According to surveys, exhaustive exercise (more than $85 \%$ of VO2 max) and long term exercise have caused change in amount of cytokine. However, moderate exercise had no significant change in the amount of cytokine (20-22). In this context, Duzova et al. stated that the long-term or extreme activities had increased the amounts of IL-17, whereas those who had performed short-term or moderate activities had no increase (21). Furthermore in our study, there was presumably no significant change in IL17 levels because of the short duration of the exercise. Golzari et al. found out that using an 8-week combined program including warm-up, stretching exercises, strength exercises, aerobic exercise and relaxation program at the end of two sessions, did not increase the level of IL-17 and even in some cases there was reduction because of low levels of exercise intensity, as 80 percent of their training program, had been planned with low-intensity exercise
(22). As it is clear in Table 1, the amount of IL-17 in various stages of research fields such as handball and volleyball has increased before, after, two hours. The increase in handball athletes after practice and in volleyball 2 hours after was greater. In Sepak-Takraw athletes, the amount of these cytokine levels decreased after increasing postexercise but increased in mountaineers after the initial fall after the workout. Thus in mountaineers this variable was associated with an increase in the two hours after exercise, although these changes were not significant.

The other point was the low levels of this cytokine in Sepak-Takraw and climbing in pre-exercise compared to handball and volleyball. Volleyball athletes had the highest increase at 2 hours after exercise and mountaineers had the greatest reduction in the next stage after training but these changes were not significant. Therefore relationship between components of evaluation of the immune system and their link to other organ systems should be investigated in the future. The role of short term intense or prolonged aerobic and exhaustive exercises on serum IL-17 and the role of this cytokine in inflammation of skeletal muscle need to be investigated in future.

The results of this study indicate that the amount of serum IL-17 measurement in all four groups of studied athletes has not significantly changed compared to those of pre-exercise. Further complementary studies need to be done to investigate the role of intense exercise on the serum level of IL-17 in other intense exercises.

\section{Acknowledgements}

The authors hereby, especially thank all dear colleagues of Urmia University Department of sport physiology for 
nice co-operation and support. Also this work has been part of a student thesis from Urmia University, Department of sport physiology.

\section{Funding/Support}

This study was financially supported by Department of Sport Physiology, Urmia University of Medical Sciences.

\section{References}

1. Hoffman-Goetz L, Pedersen BK. Exercise and the immune system: a model of the stress response? Immunol Today. 1994;15(8):382-7.

2. Nieman DC. Exercise immunology: future directions for research related to athletes, nutrition, and the elderly. Int I Sports Med. 2000;21 Suppl 1:S61-8.

3. Nieman DC. Exercise immunology: practical applications. Int Sports Med.1997;18 Suppl 1:S91-100.

4. Malm C. Exercise immunology: the current state of man and mouse. Sports Med. 2004;34(9):555-66.

5. Pedersen BK, Nieman DC. Exercise immunology: integration and regulation. Immunol Today.1998;19(5):204-6.

6. Shephard RJ. Special feature for the Olympics: effects of exercise on the immune system: overview of the epidemiology of exercise immunology. Immunol Cell Biol. 2000;78(5):485-95.

7. Timmons BW. Paediatric exercise immunology: health and clinical applications. Exerc Immunol Rev. 2005;11:108-44.

8. Smith JA. Guidelines, standards, and perspectives in exercise im munology. Med Sci Sports Exerc. 1995;27(4):497-506.

9. Bernstein E, Kaye D, Abrutyn E, Gross P, Dorfman M, Murasko DM. Immune response to influenza vaccination in a large healthy elderly population. Vaccine. 1999;17(1):82-94.

10. Ramson R, Jurimae J, Jurimae T, Maestu J. The influence of increased training volume on cytokines and ghrelin concentration in college level male rowers. Eur J Appl Physiol. 2008;104(5):839-46.

11. Robson-Ansley PJ, Blannin A, Gleeson M. Elevated plasma in terleukin-6 levels in trained male triathletes following an acute period of intense interval training. Eur J Appl Physiol.
2007:99(4):353-60.

12. Maier SF, Watkins LR. Cytokines for psychologists: implications of bidirectional immune-to-brain communication for understanding behavior, mood, and cognition. Psychol Rev. 1998;105(1):83-107.

13. Albanesi C, Cavani A, Girolomoni G. IL-17 is produced by nickelspecific $\mathrm{T}$ lymphocytes and regulates ICAM-1 expression and chemokine production in human keratinocytes: synergistic or antagonist effects with IFN-gamma and TNF-alpha. J Immunol. 1999;162(1):494-502.

14. Melzer N, Meuth SG, Torres-Salazar D, Bittner S, Zozulya AL, Weidenfeller $\mathrm{C}$, et al. A beta-lactam antibiotic dampens excitotoxic inflammatory CNS damage in a mouse model of multiple sclerosis. PLoS One. 2008;3(9)

15. Moseley TA, Haudenschild DR, Rose L, Reddi AH. Interleukin-17 family and IL-17 receptors. Cytokine Growth Factor Rev. 2003;14(2):155-74.

16. Zhu X, Mulcahy LA, Mohammed RA, Lee AH, Franks HA, Kilpatrick L, et al. IL-17 expression by breast-cancer-associated macrophages: IL-17 promotes invasiveness of breast cancer cell lines. Breast Cancer Res. 2008;10(6):R95.

17. Pischon T, Hu FB, Rexrode KM, Girman CJ, Manson JE, Rimm EB. Inflammation, the metabolic syndrome, and risk of coronary heart disease in women and men. Atherosclerosis. 2008;197(1):392-9.

18. Shoelson SE, Lee J, Goldfine AB. Inflammation and insulin resistance. JClin Invest. 2006;116(7):1793-801.

19. Tsatsanis C, Androulidaki A, Venihaki M, Margioris AN. Signalling networks regulating cyclooxygenase-2. Int J Biochem Cell Biol. 2006;38(10):1654-61.

20. Chang SH, Dong C. A novel heterodimeric cytokine consisting of IL-17 and IL-17F regulates inflammatory responses. Cell Res. 2007;17(5):435-40.

21. Duzova H, Karakoc Y, Emre MH, Dogan ZY, Kilinc E. Effects of Acute Moderate and Strenuous Exercise Bouts on IL-17 Production and Inflammatory Response in Trained Rats.J Sports Sci Med. 2009;8(2):219-24.

22. Golzari Z, Shabkhiz F, Soudi S, Kordi MR, Hashemi SM. Combined exercise training reduces IFN-gamma and IL-17 levels in the plasma and the supernatant of peripheral blood mononuclear cells in women with multiple sclerosis. Int Immunopharmacol. 2010;10(11):1415-9. 